\title{
Longitudinal Change in Peripheral Quantitative Computed Tomography Assessment in Older Adults: The Hertfordshire Cohort Study
}

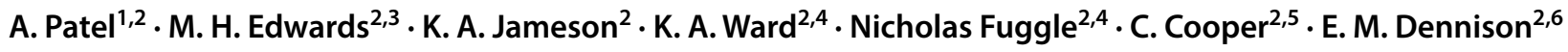

Received: 13 April 2018 / Accepted: 11 June 2018 / Published online: 21 June 2018

(c) The Author(s) 2018

\begin{abstract}
There are few longitudinal data on change in bone structure and muscle mass, strength and function in later life. We report these, and consider bone-muscle interrelationships in older men and women. We studied 188 men and 166 women from the Hertfordshire Cohort Study, who underwent peripheral quantitative computed tomography (pQCT) of the radius and tibia in 2004-2005 and then again in 2011-2012. Grip strength and gait speed were also assessed at both timepoints. Percentage change per year was calculated for grip strength, gait speed, muscle cross-sectional area (mCSA), fat cross-sectional area (fCSA) and diaphyseal bone parameters [total area (Tt.Ar), cortical area (Ct.Ar), cortical density (cBMD) and trabecular density (tBMD)]. The mean (SD) age of men and women at baseline was 68.9 (2.5) and 69.2 (2.6) years, respectively. Rates of muscle area and strength loss did not differ by sex. Tt.Ar increased with age and faster in men [mean (SD) 1.78

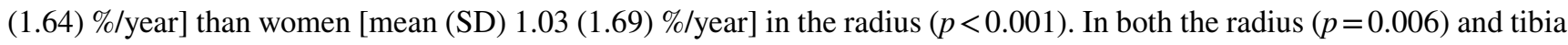
$(p<0.001)$, Ct.Ar reduced more rapidly in women than men. Change in Ct.Ar was associated with change in muscle area in the corresponding limb (radius; men: regression coefficient $0.36,95 \%$ CI $0.20-0.52, p<0.001$; tibia; men: regression coefficient $0.14,95 \%$ CI $0.00-0.27, p=0.043$, women: regression coefficient $0.16,95 \%$ CI $0.01-0.30, p=0.032$ ). We have demonstrated that muscle strength and function decrease faster than muscle mass and have provided further evidence that changes in bone structure with age differ by sex.
\end{abstract}

Keywords Peripheral quantitative computed tomography $(\mathrm{pQCT}) \cdot$ Epidemiology $\cdot$ Muscle $\cdot$ Bone mineral density $\cdot$ Bone parameters

\section{Introduction}

Electronic supplementary material The online version of this article (https://doi.org/10.1007/s00223-018-0442-0) contains supplementary material, which is available to authorized users.

E. M. Dennison

emd@mrc.soton.ac.uk

1 University Hospital Southampton NHS Foundation Trust, Southampton, UK

2 MRC Lifecourse Epidemiology Unit, University of Southampton, Southampton, UK

3 Portsmouth Hospitals NHS Trust, Portsmouth, UK

4 MRC Elsie Widdowson Laboratory, Cambridge, UK

5 NIHR Musculoskeletal Biomedical Research Unit, Nuffield Department of Orthopaedics, Rheumatology and Musculoskeletal Sciences, University of Oxford, Oxford, UK

6 Victoria University, Wellington, New Zealand
In recent years, new techniques to assess bone mass and strength have been developed. One such technique is peripheral quantitative computer tomography (pQCT) [1]. This technique was introduced some years ago as a method for assessing 3D bone structure. In particular, it has the ability to measure volumetric bone mineral density (vBMD) and can estimate trabecular and cortical compartments of bone, as well as provide some information about the surrounding muscles [2]. With the above in mind, pQCT is thought to have the potential to capture novel aspects of bone and muscle geometry that may contribute to fracture risk; however, few longitudinal data are available for change in bone and muscle compartments using this technique.

The propensity to fracture is dependent upon the mechanical strength of a bone balanced against the forces 
it endures. Bones adapt to mechanical loads generated by mechanical usage, such as muscle contraction [3], implying the direct role of muscle on bone structure and strength. There are few longitudinal studies that have focused on associations between muscle measurements and changes in bone mineral content, density, organisation and structure [4-6]. Since mechanical loading preferentially increases bone size over mineral content and density [7], neglecting the assessment of bone structure may lead to an incomplete understanding of the influence of muscle loading on bone ageing. Furthermore, to date, few studies have investigated the relationship between muscle strength or physical performance and bone structure, and those investigating these associations often fail to consider potential confounding factors such as bone mass index, body size, diet and lifestyle [8-11]. We previously performed a cross-sectional analysis of the relationships between bone mass and strength with muscle mass, strength and function among participants from the Hertfordshire Cohort Study [12], reporting that muscle size was positively associated with radial bone size and strength in both sexes, with relationships persisting after adjustment for confounders. While grip strength showed similar associations with bone size and strength in both sexes, these were substantially attenuated after similar adjustment. Consistent relationships between gait speed and bone structure were not seen. In this study population, men had bigger muscles in both their upper and lower limbs, stronger grip strength and walked faster than women and as expected in both the radius and tibia, measures of bone size and density were higher in men than women.

Musculoskeletal ageing comprises loss of muscle mass and function, and perturbation of bone microarchitecture, resulting in increased propensity to fracture with low trauma, with its attendant associated morbidity and mortality. Therefore, the purpose of this longitudinal study was to build on previous work and document change in muscle size, strength and function and bone structure and mass with age, and to investigate the interrelationships between muscle size, strength or physical performance with changes in bone structure in a well-phenotyped cohort of older men and women.

\section{Methods}

The Hertfordshire Cohort Study (HCS) is a populationbased cohort study in the UK which was designed to examine the relationship between growth in infancy and the subsequent risk of adult disease. Study design and recruitment have been described in detail previously [13]. In brief, we traced men and women born between 1931 and 1939 in Hertfordshire and who still lived there in
1998-2003 when a nurse-administered questionnaire was carried out. In 2004-2005, 437 men and 447 women from the geographical area of East Hertfordshire were invited to a follow-up study to examine their musculoskeletal health. Of these, 322 men (65\%) and 320 women (68\%) agreed to participate. In 2011-2012, participants were invited to a second follow-up study to investigate change in their musculoskeletal health. Of the original 642 follow-up participants, 443 (222 men and 221 women) re-attended.

In 2004-2005, a detailed questionnaire was administered by a trained research nurse to gather information on lifestyle, medical history, cigarette smoking and alcohol consumption. In women, years since menopause and use of oestrogen replacement therapy were also obtained. A standardised activity score, ranging $0-100$, was calculated based on responses to questions about the frequency of gardening, housework, climbing stairs and carrying loads in a typical week. Higher scores indicate a greater level of activity. Dietary calcium and socioeconomic status data had been collected at the initial questionnaire in 1998-2003. Height was measured to the nearest $0.1 \mathrm{~cm}$ and weight to the nearest $0.1 \mathrm{~kg}$ on a SECA floor scale (Chasmors Ltd, London, UK). Body mass index (BMI) was calculated as weight divided by height ${ }^{2}\left(\mathrm{~kg} / \mathrm{m}^{2}\right)$. Grip strength was measured three times in each hand using a Jamar hand-held isokinetic dynamometer using a standardised protocol [14]. The maximum value was used in analyses. Gait speed was quantified from the time taken to complete a 3-m walk test [15]. These anthropometric determinants were measured in 2004-2005 and again in 2011-2012.

In 2004-5, radial and tibial (non-dominant side) pQCT scans (Stratec 2000XL instrument, version 6.00) were performed on 276 men and 292 women. If the non-dominant side had previously sustained a fracture, the other side was scanned instead. During the second follow-up study in 2011-2012, 184 men and 166 women underwent repeat pQCT scans.

The radial length was measured from the distal end of the ulna styloid to the tip of the olecranon in millimetres $(\mathrm{mm})$. The tibial length was measured from the prominence of the medial malleolus to the tibial plate $(\mathrm{mm})$. Radial and tibial scout views identified measurement reference lines at the cortical end plates. Two slices were taken from the radial scan (4 and 66\%), as well as forearm crosssectional area of muscle (mCSA) and fat (fCSA). Three slices were taken from the tibial scan (4, 14 and 38\%), as wSA and fCSA.. Muscle size analysis by pQCT has been found to be valid and reliable [16].

Trabecular parameters were measured distally ( $4 \%$ radius and $4 \%$ tibia) and cortical parameters were measured in the mid-shaft (radius, 66\%; tibia, 14\%) The following measurements were taken from the radius and tibia: total bone area 
(Tt.Ar), total mass, trabecular bone mineral density (tBMD), cortical bone mineral density (cBMD) and cortical bone area (Ct.Ar). Short-term measurement precision error ranged from $0.88 \%$ (total tibial density, $4 \%$ slice) to $8.8 \%$ (total radial area, $66 \%$ slice), but was typically between $1-3 \%$. These figures were obtained by 20 volunteers who were part of the study undergoing two scans on the same day, with limb repositioning between examinations.

For all scans, a threshold of $280 \mathrm{mg} / \mathrm{cm}^{3}$ was used to separate the bone from the soft tissue background. Once separated, the default peeling algorithm was applied to the distal $4 \%$ scans to separate trabecular bone. With this peeling, $55 \%$ of the outer bone area was concentrically separated and defined as cortical and subcortical; the remaining $45 \%$ was defined as trabecular bone. For proximal scan locations, the default threshold of $710 \mathrm{mg} / \mathrm{cm}^{3}$ was used to separate cortical bone. Muscle CSA at the forearm and calf was derived using the default analysis steps that utilise various threshold and edge tracking settings to segment muscle from subcutaneous fat.

Ethical permission for the study was granted by the East and North Hertfordshire Ethical Committees. All participants gave written informed consent. Study participants who were taking drugs that are known to alter bone metabolism (e.g. Bisphosphonates) were excluded from the study. However, women who were taking hormone replacement therapy (HRT) were allowed to participate.

Variables were assessed for normality and transformed where necessary using the Fisher-Yates rank-based inverse normal transformation to create $z$-scores. Descriptive statistics for continuous variables were expressed as mean and standard deviation (SD) or median and interquartile range (IQR). Categorical variables were expressed as frequency and percentage (\%). Differences between men and women were assessed using Student's $t$ tests, Mann-Whitney tests or Pearson's $\chi^{2}$ tests, as appropriate. Linear regression analyses were used to examine the associations between longitudinal changes in muscle area and strength and change in bone structure over time. The regression analyses were undertaken with and without adjustment for the following lifestyle confounders: age, BMI, social class, smoker status, alcohol consumption, physical activity, dietary calcium intake and years since menopause and HRT use in women. All confounders were associated with at least one bone and muscle variable. None of the subjects were on bisphosphonate therapy at baseline but 41 subjects ( 9 men, 32 women) were receiving these therapies at follow-up; analyses were performed including and excluding these 41 people but excluding these people made little difference to the results. Similarly, we performed analyses including and excluding individuals on calcium or vitamin D supplements but again these adjustments made little difference to the results. Analyses were performed in men and women separately as we were interested in differing relationships between the two sexes, as has been observed in cross-sectional work [12]. However, we also tested for gender interactions; there was only one significant interaction (for change in CMA against tibial trabecular density). A $p$ value of $\leq 0.05$ was considered to be significant for all analysis. Statistical analyses were performed using STATA, version 14.

\section{Results}

The baseline characteristics of the study population are displayed in Table 1; the mean age was 68.9 and 69.2 years in men and women, respectively. All women were postmenopausal. The mean body mass index score for women was $27.4 \mathrm{~kg} / \mathrm{m}^{2}$, compared to $27.0 \mathrm{~kg} / \mathrm{m}^{2}$ for men. Among men, $60.4 \%$ were either current or ex-smokers, compared with only $34.8 \%$ of women $(p<0.001)$. Men consumed more alcohol compared to women $(p<0.001)$. At baseline, men had larger muscles in both their upper and lower limbs $(p<0.001)$, stronger grip strength $(p<0.001)$ and walked faster than women $(p=0.010)$. In contrast, women had greater amounts of fat in their upper and lower limbs than men $(p<0.001)$. Additionally, at baseline, men had greater bone mass, density, size and strength in the radius, than women $(p<0.001)$. Similar sex-differences were found in the tibia bone parameters $(p<0.001)$.

Longitudinal changes in muscle and bone parameters are summarised in Table 2, with change in muscle mass, strength and function also displayed in Fig. 1. Muscle strength and function decreased faster than muscle mass, as displayed in the figure. As expected, over time, men and women's forearm and calf mCSA reduced. However, the rate of loss did not differ significantly by sex. In both sexes, the rate of loss of grip strength and gait speed was over $2 \%$ per year, whereas muscle size reduced at a slower rate. Figure 1 provides a graphical representation of the proportionate change in men and women of muscle area at the forearm and calf, grip strength and gait speed. The rate of increase in forearm and calf fCSA differed by sex, affecting men more than women [forearm; men: mean (SD) 1.25 (4.84) \%/year, women: mean (SD) 0.17 (2.15) \%/year, calf: men: mean (SD) 3.62 (20.5) \%/year, women: mean (SD) 0.57 (2.65) \%/ year]. However, only the rate of increase in forearm fCSA reached statistical significance $(p=0.010)$.

In both the radius and tibia, total bone mass and trabecular density tended to reduce at a greater rate in women than men $(p<0.10)$. Proximally, total area had a tendency to increase over time, showing positive values in both sexes. The rate of increase was significantly higher in men [mean (SD) 1.78 (1.64) \%/year] than women [mean (SD) 1.03 (1.69) \%/year] in the radius $(p<0.001)$. The rate of loss of cortical area was significantly greater in women than men 
Table 1 Baseline characteristics of the population

\begin{tabular}{|c|c|c|c|}
\hline & Mean (SD) & Mean (SD) & \\
\hline & $\operatorname{Men}(n=184)$ & Women $(n=166)$ & $p$ value \\
\hline Age (years) & $68.9(2.5)$ & $69.2(2.6)$ & 0.412 \\
\hline BMI $\left(\mathrm{kg} / \mathrm{m}^{2}\right)$ & $27.0(3.4)$ & $27.4(4.5)$ & 0.333 \\
\hline \multirow[t]{2}{*}{ Alcohol intake (units/week) } & Median (IQR) & Median (IQR) & \\
\hline & $9.2(2.8-17.8)$ & $1.5(0.0,4.8)$ & $<0.001$ \\
\hline Smoker status & $N(\%)$ & $N(\%)$ & \\
\hline Never & $73(39.7)$ & $107(65.2)$ & $<0.001$ \\
\hline Ex & $98(53.3)$ & $49(29.9)$ & \\
\hline Current & $13(7.1)$ & $8(4.9)$ & \\
\hline Muscle parameters & Mean (SD) & Mean (SD) & \\
\hline Forearm area $\left(\mathrm{mm}^{2}\right)$ & $4019(506)$ & $2564(351)$ & $<0.001$ \\
\hline Calf area $\left(\mathrm{mm}^{2}\right)$ & 8125 (1199) & $6299(954)$ & $<0.001$ \\
\hline Grip strength (kg) & $43.1(8.0)$ & $25.2(6.0)$ & $<0.001$ \\
\hline Gait speed $(\mathrm{m} / \mathrm{s})$ & $0.94(0.16)$ & $0.89(0.16)$ & 0.010 \\
\hline Fat parameters & Mean (SD) & Mean (SD) & \\
\hline Forearm fat area $\left(\mathrm{mm}^{2}\right)$ & $1020(370)$ & $1749(720)$ & $<0.001$ \\
\hline \multirow[t]{2}{*}{ Calf fat area $\left(\mathrm{mm}^{2}\right)$} & $1718(664)$ & $3280(1953)$ & $<0.001$ \\
\hline & Mean (SD) & Mean (SD) & \\
\hline \multicolumn{4}{|l|}{ Bone parameters (radius) } \\
\hline $4 \%$ total mass $\left(\mathrm{mg} \mathrm{mm}^{-1}\right)$ & $1.63(0.25)$ & $1.01(0.18)$ & $<0.001$ \\
\hline $4 \%$ trabecular BMD $\left(\mathrm{mg} / \mathrm{cm}^{3}\right)$ & $210(37)$ & $176(44)$ & $<0.001$ \\
\hline $66 \%$ total area $\left(\mathrm{mm}^{2}\right)$ & $181(26)$ & $131(20)$ & $<0.001$ \\
\hline $66 \%$ cortical area $\left(\mathrm{mm}^{2}\right)$ & $101(14)$ & $64(11)$ & $<0.001$ \\
\hline $66 \%$ cortical BMD $\left(\mathrm{mg} / \mathrm{cm}^{3}\right)$ & $1120(36)$ & $1099(47)$ & $<0.001$ \\
\hline \multicolumn{4}{|l|}{ Bone parameters (tibia) } \\
\hline $4 \%$ total mass $\left(\mathrm{mg} \mathrm{mm}^{-1}\right)$ & $4.24(0.55)$ & $2.97(0.46)$ & $<0.001$ \\
\hline $4 \%$ trabecular BMD $\left(\mathrm{mg} / \mathrm{cm}^{3}\right)$ & $241(37)$ & $222(43)$ & $<0.001$ \\
\hline $14 \%$ total area $\left(\mathrm{mm}^{2}\right)$ & $575(81)$ & $472(60)$ & $<0.001$ \\
\hline $14 \%$ cortical area $\left(\mathrm{mm}^{2}\right)$ & $201(25)$ & $141(22)$ & $<0.001$ \\
\hline $14 \%$ cortical BMD $\left(\mathrm{mg} / \mathrm{cm}^{3}\right)$ & $1101(29)$ & $1047(50)$ & $<0.001$ \\
\hline
\end{tabular}

Table 2 Change in muscle, fat and bone parameters with time in the cohort

\begin{tabular}{|c|c|c|c|c|c|c|}
\hline \multirow[t]{2}{*}{ Muscle parameters } & \multicolumn{3}{|l|}{ Forearm } & \multicolumn{3}{|l|}{ Calf } \\
\hline & $\begin{array}{l}\text { Men } \\
\text { Mean (SD) }\end{array}$ & $\begin{array}{l}\text { Women } \\
\text { Mean (SD) }\end{array}$ & $p$ value & $\begin{array}{l}\text { Men } \\
\text { Mean (SD) }\end{array}$ & $\begin{array}{l}\text { Women } \\
\text { Mean (SD) }\end{array}$ & $p$ value \\
\hline Muscle area (\%/year) & $-0.75(0.87)$ & $-0.67(0.86)$ & 0.360 & $-0.34(1.15)$ & $-0.17(1.09)$ & 0.175 \\
\hline Fat area (\%/year) & $1.25(4.84)$ & $0.17(2.15)$ & 0.010 & $3.62(20.48)$ & $0.57(2.65)$ & 0.069 \\
\hline Grip strength (\%/year) & $-2.26(2.02)$ & $-2.00(2.94)$ & 0.323 & & & \\
\hline Gait speed (\%/year) & & & & $-2.38(2.73)$ & $-2.67(2.96)$ & 0.354 \\
\hline $4 \%$ total mass (\%/year) & $-0.50(0.84)$ & $-0.69(0.96)$ & 0.055 & $-0.40(0.78)$ & $-0.59(0.64)$ & 0.015 \\
\hline $4 \%$ trabecular BMD (\%/year) & $-0.22(1.08)$ & $-0.43(1.25)$ & 0.097 & $-0.32(0.77)$ & $-0.47(0.75)$ & 0.065 \\
\hline $66 / 14 \%$ total area (\%/year) & $1.78(1.64)$ & $1.03(1.69)$ & $<0.001$ & $0.38(0.41)$ & $0.37(0.30)$ & 0.762 \\
\hline $66 / 14 \%$ cortical area (\%/year) & $-0.24(0.92)$ & $-0.56(1.16)$ & 0.006 & $-0.37(0.70)$ & $-0.87(1.08)$ & $<0.001$ \\
\hline $66 / 14 \%$ cortical BMD (\%/year) & $-0.29(0.28)$ & $-0.15(0.39)$ & 0.001 & $-0.36(0.24)$ & $-0.42(0.37)$ & 0.056 \\
\hline
\end{tabular}




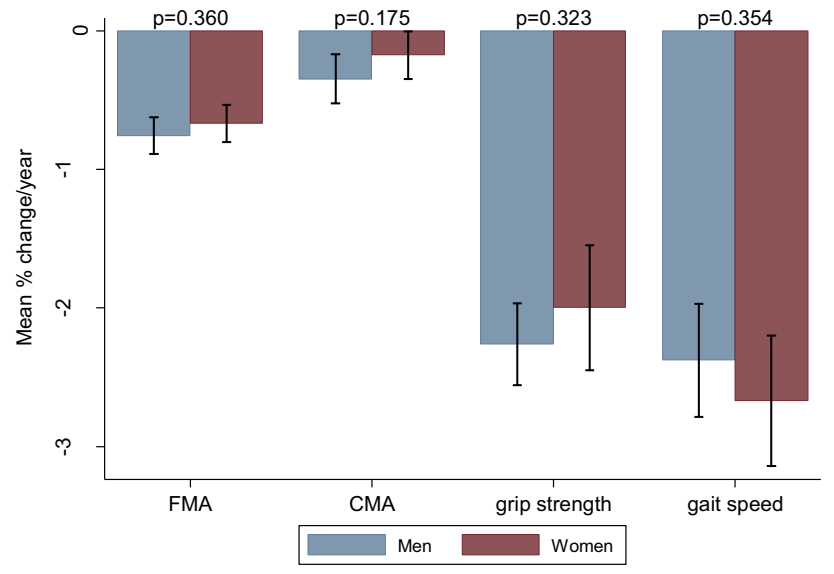

Fig. 1 Percentage change in men and women of muscle area at the forearm (FMA) and calf (CMA); grip strength; gait speed

in both the radius $(p=0.006)$ and tibia $(p<0.001)$. In the radius, cortical density decreased at a greater rate for men than for women $(p=0.001)$.

In general, changes in cortical bone geometry, not cortical BMD, were more strongly associated with changes in muscle mass and function, particularly at the radius (Table 3). In men, positive associations were seen between change in forearm muscle area and changes in radial total mass $z$-score (regression coefficient $0.30,95 \%$ CI $0.14-0.47, p<0.001$ ) distally, radial Ct.Ar $z$-score (regression coefficient 0.36 , 95\% CI $0.20-0.52, p<0.001$ ) and cBMD $z$-score (regression coefficient $0.18,95 \%$ CI $0.01-0.35, p=0.034$ ) in the midshaft, although the latter was not maintained after adjustment for demographic and lifestyle factors. No associations were seen in women. Change in calf muscle area was associated with changes in tibial Ct.Ar $z$-score, in both men (regression coefficient $0.14,95 \%$ CI $0.00-0.27, p=0.043$ ) and women (regression coefficient $0.16,95 \%$ CI $0.01-0.30$, $p=0.032$ ), though the association did not remain significant in men after adjustment for demographic and lifestyle factors. In women, there were also positive associations between change in calf muscle area and total mass $z$-score (regression coefficient $0.19,95 \%$ CI $0.05-0.33, p=0.007$ ) and tBMD $z$-score (regression coefficient $0.25,95 \% \mathrm{CI}$ $0.12-0.39, p<0.001)$.

Positive associations were found between change in grip strength and change in forearm mCSA ( $z$-score) in both sexes (men: regression coefficient $0.11,95 \%$ CI $0.03-0.18$, $p=0.005$, women: regression coefficient: $0.06,95 \% \mathrm{CI}$ $0.01-0.11, p=0.015)$. In women, change in gait speed was also associated with change in calf mCSA $z$-score (regression coefficient $0.07,95 \%$ CI $0.01-0.12, p=0.012$ ). In both men and women, a change in gait speed was associated with a change in calf fCSA $z$-score (men: regression coefficient $-0.06,95 \% \mathrm{CI}-0.12$ to $-0.01, p=0.029$; women: regression coefficient $-0.05,95 \% \mathrm{CI}-0.11$ to $-0.00, p=0.045$ ), though this association did not remain in women after adjustment for demographic and lifestyle factors.

Change in grip strength was associated with change in total radial mass $z$-score in men (regression coefficient $0.09,95 \%$ CI $0.01-0.16, p=0.025$ ) but not when demographic and lifestyle factors were adjusted for. Change in grip strength was not associated with any other changes in radial size or bone mineral density in either sex. In men, change in grip strength was associated with change in tibia diaphysis total area $z$-score (regression coefficient $0.10,95 \%$ CI $0.02-0.17, p=0.016$ ) and tibial mass $z$-score but only when demographic and lifestyle factors were adjusted for (regression coefficient $0.10,95 \%$ CI $0.02-0.19, p=0.022$ ).
Table 3 Interrelationships between change in muscle and bone parameters

\begin{tabular}{|c|c|c|c|c|c|c|}
\hline & \multicolumn{2}{|c|}{$\begin{array}{l}\text { Change in forearm } \\
\text { muscle area }\end{array}$} & \multicolumn{2}{|c|}{$\begin{array}{l}\text { Change in grip } \\
\text { strength }\end{array}$} & \multicolumn{2}{|c|}{$\begin{array}{l}\text { Change in calf } \\
\text { muscle area }\end{array}$} \\
\hline & Men & Women & Men & Women & Men & Women \\
\hline \multicolumn{7}{|l|}{ Radius } \\
\hline Change in total mass $4 \%$ site & $0.30 * *$ & 0.09 & $0.09 *$ & 0.02 & & \\
\hline Change in trabecular density $4 \%$ site & 0.12 & 0.03 & 0.03 & 0.01 & & \\
\hline Change in total area $66 \%$ site & -0.02 & 0.05 & 0.05 & -0.01 & & \\
\hline Change in cortical area $66 \%$ site & $0.36^{* *}$ & 0.04 & 0.04 & -0.03 & & \\
\hline Change in cortical density $66 \%$ site & $0.18 *$ & 0.06 & 0.06 & 0.02 & & \\
\hline \multicolumn{7}{|l|}{ Tibia } \\
\hline Change in total mass $4 \%$ site & & & 0.03 & -0.01 & 0.06 & $0.19 *$ \\
\hline Change in trabecular density $4 \%$ site & & & $0.06 *$ & -0.02 & -0.02 & $0.25 * *$ \\
\hline Change in total area $14 \%$ site & & & 0.03 & 0.00 & -0.01 & -0.13 \\
\hline Change in cortical area $14 \%$ site & & & 0.01 & -0.01 & $0.14^{*}$ & $0.16^{*}$ \\
\hline Change in cortical density $14 \%$ site & & & -0.01 & 0.02 & 0.06 & 0.13 \\
\hline
\end{tabular}

${ }^{*} p<0.05,{ }^{* *} p<0.001$ 
In contrast, no relationship was identified between change in grip strength and change in tibial size or density in women.

\section{Discussion}

In this study, we report change in bone microarchitecture and muscle mass, strength and function with age in a community dwelling cohort of older adults. We have demonstrated that muscle strength and function decrease at a faster rate than muscle mass and furthermore have provided further evidence that changes in bone structure with age differ by sex. Cortical area consistently decreased more rapidly in women than men, whereas total bone area consistently increased more rapidly in men than women. In contrast, rates of loss of muscle parameters were similar in both sexes. Additionally, we have shown that there were positive associations between changes in muscle area and cortical area in both men and women. Finally, we have shown that changes in grip strength and gait speed were strongly associated with changes in muscle area in both sexes, and changes in bone size in men, but not women are in keeping with previous analyses [12].

The associations between changes both in muscle and bone size can be explained in several ways. First, the mechanostat hypothesis [3] states that bone adapts to changes in muscle in order to maintain appropriate strength to not fail during muscular loading. This is through activation of osteocytes which are able to sense strain; they either promote osteogenesis leading to bone formation or decrease bone resorption leading to reduced bone loss [17]. Second, we must acknowledge the various effects that genetic, nonmechanical (e.g. nutrition) and hormonal factors have on the musculoskeletal system, which can either directly or indirectly impact both muscle and bone development [18-21]. Third, physical activity can affect both muscle size and strength and bone structure. Finally, there is evidence supporting associations between birth weight and both bone health [22] and muscle function [23] in later life.

This study demonstrated that changes in grip strength and gait speed were strongly associated with changes in muscle size in both sexes, and changes in bone size in men, but not women. To our knowledge, the relationship between changes in physical performance as measured by grip strength and gait speed and changes in bone structure have not been previously investigated. However, a similar study, STRAMBO [24], defined physical performance by chair rises, static and dynamic balance and found that participants who scored poorly on one of the tests had significantly lower cortical bone area as assessed by high-resolution pQCT. These results are in keeping with our study's finding although our assessment of physical performance employed different techniques.
Our analysis did not reveal consistent relationships between both changes in muscle area and strength and changes in bone mineral density (tBMD, cBMD) in weightbearing and non-weight-bearing limbs. This is consistent with animal studies [25] that have shown cortical bone adapts its strength to mechanical loading by preferentially increasing bone size rather than bone mineral density, which is in keeping with our findings.

The main strength of this study was the population investigated. The individuals recruited were a non-selective community dwelling population who were born in Hertfordshire, and continued to live there at the age of 60-75 years. HCS characteristic and mortality patterns have previously been demonstrated to be similar to England [26]; thus generalisation to the wider population of older adults can be made. However, there are some limitations of this study. While not a true limitation, grip strength was taken as the highest of six measurements (three on the dominant side and three on the non-dominant side). Naturally, one would expect the maximum grip strength measurement to be recorded from the dominant side. Bone parameters were assessed on the non-dominant side, therefore this may attenuate the associations seen between grip strength and bone parameters to a degree. However, previous literature has reported the difference between grip strength in the dominant and nondominant limbs to be small and relatively consistent [27]. Furthermore, the radius and tibia are not completely circular in cross section, therefore the manufacturer recommends the use of a circular ring model assumption in analysis of pQCT images. The alternative strategy would be to use an iterative contour detection method to provide a direct measurement, based on the true shape of the bone. However, because of the limited spatial resolution of pQCT, the pitfall in using an iterative approach is the failure to produce a measurement that can be utilised in an appreciable portion of individuals. It would be interesting to include body composition from DXA in our analyses but unfortunately these data were not available at the time points in question. Finally, we also recognise the limitations associated with self-reported physical activity, dietary calcium, smoking status and alcohol consumption.

In conclusion, we have demonstrated that changes in bone structure with age differ by sex. Additionally, we have shown that longitudinal changes in muscle size, in both upper and lower limbs, was strongly associated with longitudinal changes in bone size, in both men and women. This suggests that interventions to maintain muscle mass may help to ameliorate the age-related deterioration in bone health.

Acknowledgements The Hertfordshire Cohort Study (HCS) was supported by the Medical Research Council of Great Britain and Arthritis Research UK. We thank all of the men and women who took part in the HCS and the HCS Research Staff. 
Author Contributions EMD and $\mathrm{CC}$ were involved in data acquisition. AP drafted the manuscript with input from KAJ, MHE, KW, NF, CC and EMD. All authors contributed to the analysis and interpretation of the data, revised the manuscript critically for important intellectual content and approved the final version of the submitted manuscript. AP accepts responsibility for the integrity of the data analysis.

\section{Compliance with Ethical Standards}

Conflict of interest Professor C Cooper has received consultancy fees/ honoraria from Servier; Eli Lilly; Merck; Amgen; Alliance; Novartis; Medtronic; GSK; Roche. A. Patel, M. H. Edwards, K. A. Jameson, K. A. Ward, Nicholas Fuggle and E. Dennison declare that they have no conflict of interest.

Human and Animal Rights and Informed Consent This research was performed in accordance with the Declaration of Helsinki and was approved by the UK Health Research Authority, reference 07/ MRE01/30. All participants gave informed consent.

Open Access This article is distributed under the terms of the Creative Commons Attribution 4.0 International License (http://creativeco mmons.org/licenses/by/4.0/), which permits unrestricted use, distribution, and reproduction in any medium, provided you give appropriate credit to the original author(s) and the source, provide a link to the Creative Commons license, and indicate if changes were made.

\section{References}

1. Hung VW, Qin L, Au SK, Choy VW, Leung KS, Leung PC, Cheng JC (2004) Correlation of calcaneal QUS with pQCT measurements at distal tibial and non-weight-bearing distal radius. J Bone Miner Metab 22:486-490

2. Dennison EM, Jameson KA, Edwards MH, Denison HJ, Sayer AA, Cooper C (2014) Peripheral quantitative computed tomography measures are associated with adult fracture risk: the Hertfordshire Cohort Study. Bone 64:13-17

3. Frost HM (2003) Bone's mechanostat: a 2003 update. Anat Rec Part A 2:1081-1101

4. Segal NA, Torner JC, Yang M, Curtis JR, Felson DT, Nevitt MC (2008) Muscle mass is more strongly related to hip bone mineral density than is quadriceps strength or lower activity level in adults over age 50 year. J Clin Densitom 4:503-510

5. Blain H, Vuillemin A, Teissier A, Hanesse B, Guillemin F, Jeandel C (2001) Influence of muscle strength and body weight and composition on regional bone mineral density in healthy women aged 60 years and over. Gerontology 4:207-212

6. Sirola J, Tuppurainen M, Honkanen R, Jurvelin JS, Kroger H (2005) Associations between grip strength change and axial postmenopausal bone loss-a 10-year population-based followup study. Osteoporos Int 12:1841-1848

7. Robling AG, Castillo AB, Turner CH (2006) Biomechanical and molecular regulation of bone remodeling. Ann Rev Biomed Eng 8:455-498

8. Cousins JM, Petit MA, Paudel ML, Taylor BC, Hughes JM, Cauley JA, Zmuda JM, Cawthon PM, Ensrud KE (2010) Muscle power and physical activity are associated with bone strength in older men: the osteoporotic fractures in men study. Bone 2:205-211

9. Kaji H, Kosaka R, Yamauchi M, Kuno K, Chihara K, Sugimoto $\mathrm{T}$ (2005) Effects of age, grip strength and smoking on forearm volumetric bone mineral density and bone geometry by peripheral quantitative computed tomography: comparisons between female and male. Endocrinol J 6:659-666

10. Hasegawa Y, Schneider P, Reiners C (2001) Age, sex, and grip strength determine architectural bone parameters assessed by peripheral quantitative computed tomography (pQCT) at the human radius. J Biomech 4:497-503

11. Frank AW, Lorbergs AL, Chilibeck PD, Farthing JP, Kontulainen SA (2010) Muscle cross sectional area and grip torque contraction types are similarly related to pQCT derived bone strength indices in the radii of older healthy adults. J Musculoskelet Neuronal Interact 2:136-141

12. Edwards MH, Gregson CL, Patel HP, Jameson KA, Harvey NC, Sayer AA, Dennison EM, Cooper C (2013) Muscle size, strength and physical performance and their associations with bone structure in the Hertfordshire Cohort Study. J Bone Miner Res 11:2295-2304

13. Dennison EM, Syddall HE, Aihie Sayer A, Gilbody HJ, Cooper C (2005) Birth weight and weight at 1 year are independent determinants of bone mass in the seventh decade: the Hertfordshire Cohort Study. Paediatr Res 57:582-586

14. Bohannon RW (1986) Test-retest reliability of hand held dynamometry during a single session of strength assessment. Phys Ther 66:206-209

15. Posiadlo D, Richardson S (1991) The timed "up and go": a test of basic functional mobility for frail elderly persons. J Am Geriatr Soc 39:142-148

16. Cramer JT, Palmer IJ, Ryan ED, Herda TJ, Bemben DA, Bemben MG, Stratemeier PH (2007) Validity and reliability of a peripheral quantitative computed tomography scanner for measuring muscle cross-sectional area. Med Sci Sports Exerc 5:225-226

17. Lu TW, Taylor SJ, O'Connor JJ, Walker PS (1997) Influence of muscle activity on the forces in the femur: an in vivo study. $\mathrm{J}$ Biomech 11:1101-1106

18. Karasik D, Kiel DP (2008) Genetics of the musculoskeletal system: a pleiotropic approach. J Bone Miner Res 6:788-802

19. Urban RJ (2011) Growth hormone and testosterone: anabolic effects on muscle. Hormonal Res Paediatr 76 (Suppl 1):81-83

20. Frost HM (1998) Could some biomechanical effects of growth hormone help to explain its effects on bone formation and resorption? Bone 5:395-398

21. Hogler W, Briody J, Moore B, Lu PW, Cowell CT (2005) Effect of growth hormone therapy and puberty on bone and body composition in children with idiopathic short stature and growth hormone deficiency. Bone 5:642-650

22. Baird J, Kurshid MA, Kim M, Harvey N, Dennison E, Cooper C (2011) Does birthweight predict bone mass in adulthood? A systematic review and meta-analysis. Osteoporos Int 5:1323-1334

23. Dodds R, Denison HJ, Ntani G, Cooper R, Cooper C, Sayer AA, Baird J (2012) Birth weight and muscle strength: a systematic review and meta-analysis. J Nutr Health Aging 7:609-615

24. Blaizot S, Boutroy S, Vilayphiou N, Boonen S, Chapurlat R, Szulc P (2012) Poor bone microarchitecture in older men with impaired physical performance-the STRAMBO study. Osteoporos Int 12:2785-2796

25. Robling AG, Castillo AB, Turner CH (2006) Biomechanical and molecular regulation of bone remodelling. Annu Rev Biomed Eng 8:455-498

26. Dennison EM, Syddall HE, Aihie Sayer A, Gilbody HJ, Cooper C (2005) Birth weight, infant weight gain and cause-specific mortality: the Hertfordshire Cohort Study. Am J Epidemiol $161: 1074-1780$

27. Bechtol CO (1954) Grip test; the use of a dynamometer with adjustable handle spacings. J Bone Joint Surg 36:820-824 